Vietnam Journal of Mechanics, VAST, Vol. 42, No. 3 (2020), pp. $341-354$

DOI: https://doi.org/10.15625/0866-7136/15356

Dedicated to Professor J.N. Reddy on the Occasion of His $75^{\text {th }}$ Birthday

\title{
A MODIFIED AVERAGING OPERATOR WITH SOME APPLICATIONS
}

\author{
Anh Tay Nguyen ${ }^{1}$, N. D. Anh ${ }^{2,3, *}$ \\ ${ }^{1}$ SUNY Korea, Incheon, Republic of Korea \\ ${ }^{2}$ Institute of Mechanics, VAST, Hanoi, Vietnam \\ ${ }^{3}$ VNU University of Engineering and Technology, Hanoi, Vietnam \\ *E-mail: ndanh@imech.vast.vn
}

Received: 09 August 2020 / Published online: 27 September 2020

\begin{abstract}
The paper presents a new approach to the conventional averaging in which the role of boundary values is considered in a more detailed way. It results in a new weighted local averaging operator (WLAO) taking into account the particular role of boundary values. A remarkable feature of WLAO is that this operator contains a parameter of boundary regulation $p$ and depends on a local value $h$ of the integration domain. By varying these two parameters one can regulate the obtained approximate solutions in order to get more accurate ones. It has been shown that the combination of WLAO with Galerkin method can lead to an effective approximate tool for the buckling problem of columns and for the frequency analysis of free vibration of strongly nonlinear systems.
\end{abstract}

Keywords: weighted local averaging, Galerkin method, buckling, Euler column, free vibration, strong nonlinearity.

\section{INTRODUCTION}

In mathematics, an operator is generally a mapping or function that operates on elements of space to create elements of another space. One of the most natural and popular models of operators is integral operators that are widely and and effectively used in many fields of science and engineering in general, and in applied mathematics and mechanics, in particular. Indeed it is impossible to review the applications of integral operators in all branches of science and engineering. Well-known examples of integral operator are integral transforms such as Laplace and Fourier transforms, which are mappings between two function spaces. Integral operator is used to present functionals that are the objects of the study of the variational theory and functional analysis Oden and Reddy [1], Reddy [2]. Integral operator is an effective tool for presenting energies of a body in a deformed state, such as strain, stress energies; kinetic and potential energies for deformable body Reddy [3]. The use of integral operators allows to introduce several integral principles of mechanics, for example, variational principles that support the formulation of equations of motion or/and relationships between stresses, strains or deformations, displacements Reddy $[4,5]$. Action integral is used to formulate Hamilton's principle, which allows the derivation of differential equations of motion of mechanical systems composed of rigid and/or deformable bodies Reddy [3].

Among integral operators averaging operators form an important class because they can combine all values of a function into an average value. For example, for one-dimensional structures, the conventional averaging operator is used as a integration over total structural length. For this reason, the conventional averaging (CA) can often be cited as simple or arithmetic averaging based on the suggestion that all values have the same role for the function in question. Along with the conventional averaging, the weighted averaging (WA) is another interesting and effective approach based on the philosophy that each value has a different contribution to the function. For example weighted integrals are 
used in weighted-residual methods to extract algebraic equations from the governing differential equation in weak form for a finite model for structures Oden and Reddy [6], Reddy [7]. Both-conventional and weighted averaging-are widely used in practice but each type is more appropriate to use than the other for certain purposes and applications.

The aim of this paper is to present a simple form of weighted averaging operator taking into account the particular role of boundary values of a function. It is obtained that the connection of WA with Galerkin method can lead to an effective approximate tool for the buckling problem of columns and the frequency analysis of free vibration of nonlinear systems. With respect to analytical investigations, many attempts have been given to improve the accuracy of the first order approximate solution of Galerkin method, since this solution can usually be obtained in a simple form. Exact solutions for buckling of structural members including various cases of columns, beams, arches, rings, plates, and shells with variable cross-section, variable axial forces and different boundary conditions were given in the book by Wang C.M., Wang C.Y. and Reddy [8]. The exact solutions for buckling problem are used in this paper to check the accuracy of Galerkin method with weighted averaging. An interesting discussion about the implementation of Galerkin method for stepped beams is given recently by Elishakoff, Ankitha and Marzani [9]. The paper is organized as follows: a weighted local averaging (WLA) is presented in the second section. The application of WLA for the buckling problem of columns and the frequency analysis of free vibration of nonlinear systems is presented in Sections 3 and 4, respectively. Conclusions and further investigations are summarized in Section 5.

\section{WEIGHTED LOCAL AVERAGING WITH EMPHASIS ON BOUNDARY DOMAINS}

Let $g(x)$ is an integrable deterministic function of $x \in[0,1]$ and $h$ is a local value in $[0,1]$. The average of $g(x)$ over the interval $[0,1]$ is given by an integral as follows

$$
\langle g(x)\rangle=\int_{0}^{1} g(x) \mathrm{d} x
$$

where $\langle$.$\rangle denotes the conventional averaging operator. The average (1) is called simple average or$ arithmetic mean because all values of $g(x)$ are treated equally and assigned equal weight. However, values of $g(x)$ may be weighted for the reason that they belong to different domains of the interval $[0,1]$. It is well known that the boundary conditions play a key role in Mechanics of solids and structures Fenner and Reddy [10]. To develop this point of view, in addition to the conventional arithmetic average, we consider the following integral taken over the global domain and over some local boundary domains as follows

$$
\langle g(x), h\rangle_{p}=q \int_{0}^{1} g(x) \mathrm{d} x+p\left(\int_{0}^{h} g(x) \mathrm{d} x+\int_{1-h}^{1} g(x) \mathrm{d} x\right),
$$

where the left side is a new notation denoting the weighted local averaging (WLA) of $g(x)$ at a local value $h, q$ and $p$ are weights, second term involves values of $g(x)$ integrated in the boundary domains $[0, h]$ and $[1-h, 1]$. It is noted that the parameter $p$ is introduced in Eq. (2) to highlight a special role of $g(x)$ in boundary domains and hence $p$ can be quoted as parameter of boundary regulation. We will require that the operator (2) is conservative so one has the following equality

$$
\langle 1, h\rangle_{p}=1 \text {. }
$$

Using Eq. (2) one obtains from Eq. (3)

$$
q \int_{0}^{1} 1 \mathrm{~d} x+p\left(\int_{0}^{h} 1 \mathrm{~d} x+\int_{1-h}^{1} 1 \mathrm{~d} x\right)=q+2 p h=1,
$$

or

$$
q=1-2 p h
$$


Substituting Eq. (5) into Eq. (2) gives

$$
\langle g(x), h\rangle_{p}=(1-2 p h) \int_{0}^{1} g(x) \mathrm{d} x+p\left(\int_{0}^{h} g(x) \mathrm{d} x+\int_{1-h}^{1} g(x) \mathrm{d} x\right) .
$$

Hence, values of the function $g(x)$ are weighted for the reason that the values in the boundary regions are calculated once more and then multiple with a weight $p$. The weighted local averaging is coincident with the conventional averaging for $p=0$.

It is worth to note that WLA of $g(x)$ defined by Eq. (6) is a function of $h$. Some following properties are obtained for WLA (6):

Property. The weighted local average of $g(x)$ is equal to conventional average of $g(x)$ at three local values $h=0 ; 0.5$ and 1, i.e. one has

$$
\begin{aligned}
& \langle g(x), 0\rangle_{p}=(1-0) \int_{0}^{1} g(x) \mathrm{d} x+p\left(\int_{0}^{0} g(x) \mathrm{d} x+\int_{1}^{1} g(x) \mathrm{d} x\right)=\int_{0}^{1} g(x) \mathrm{d} x=\langle g(x)\rangle, \\
& \langle g(x), 0.5\rangle_{p}=(1-p) \int_{0}^{1} g(x) \mathrm{d} x+p\left(\int_{0}^{0.5} g(x) \mathrm{d} x+\int_{0.5}^{1} g(x) \mathrm{d} x\right)=\int_{0}^{1} g(x) \mathrm{d} x=\langle g(x)\rangle, \\
& \langle g(x), 1\rangle_{p}=(1-2 p) \int_{0}^{1} g(x) \mathrm{d} x+p\left(\int_{0}^{1} g(x) \mathrm{d} x+\int_{0}^{1} g(x) \mathrm{d} x\right)=\int_{0}^{1} g(x) \mathrm{d} x=\langle g(x)\rangle .
\end{aligned}
$$

For one term polynomial $x^{n}$ one gets

$$
\begin{aligned}
\left\langle x^{n}, h\right\rangle_{p} & =(1-2 p h) \int_{0}^{1} x^{n} \mathrm{~d} x+p\left(\int_{0}^{h} x^{n} \mathrm{~d} x+\int_{1-h}^{1} x^{n} \mathrm{~d} x\right) \\
& =(1-2 p h) \frac{1}{n+1}+p\left(\frac{h^{n+1}}{n+1}+\frac{1-(1-h)^{n+1}}{n+1}\right) \\
& =\frac{1}{n+1}\left(1+p\left(1-2 h+h^{n+1}-(1-h)^{n+1}\right)\right) .
\end{aligned}
$$

In particular one has

$$
\begin{aligned}
\langle x, h\rangle_{p} & =\frac{1}{2} \\
\left\langle x^{2}, h\right\rangle_{p} & =\frac{1}{3}(1+p h(1-h)(1-2 h)), \\
\left\langle x^{3}, h\right\rangle_{p} & =\frac{1}{4}(1+2 p h(1-h)(1-2 h)) .
\end{aligned}
$$

For harmonic functions one gets

$$
\begin{aligned}
\langle\cos (2 \pi n x), h\rangle_{p} & =(1-2 p h) \int_{0}^{1} \cos (2 \pi n x) \mathrm{d} x+p\left(\int_{0}^{h} \cos (2 \pi n x) \mathrm{d} x+\int_{1-h}^{1} \cos (2 \pi n x) \mathrm{d} x\right) \\
& =\frac{p}{\pi n} \sin 2 \pi n h, \\
\langle\sin (2 \pi n x), h\rangle_{p} & =(1-2 p h) \int_{0}^{1} \sin (2 \pi n x) \mathrm{d} x+p\left(\int_{0}^{h} \sin (2 \pi n x) \mathrm{d} x+\int_{1-h}^{1} \sin (2 \pi n x) \mathrm{d} x\right)=0 .
\end{aligned}
$$

Some graphics of $\left\langle x^{n}, h\right\rangle_{p}$ as functions of $h$, or of $p$, or of $h$ and $p$ both are shown in Figs. 1-2.

If function $g(x)$ is expanded into Taylor series

$$
g(x)=\sum_{i=0}^{\infty} g_{i} x^{i},
$$




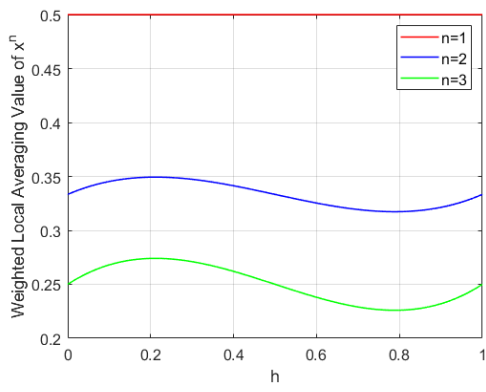

(a)

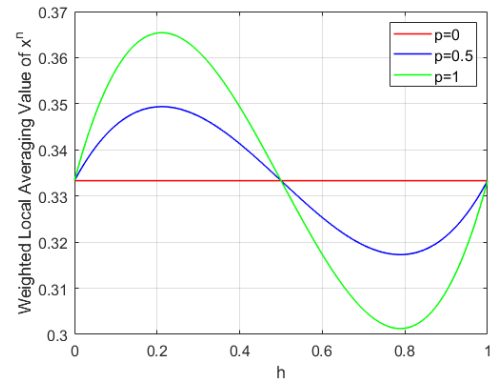

(b)

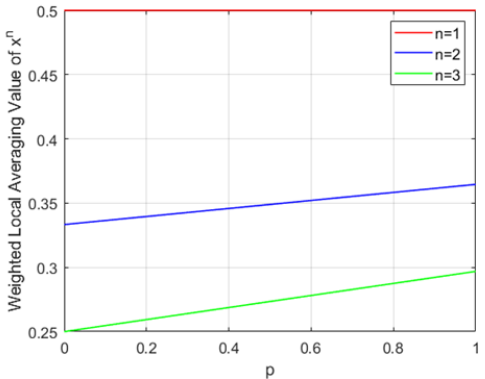

(c)

Fig. 1. WLA of $x^{n}$ as a function of (a) $h$ only and for $n=1,2,3 ; p=0.5$; (b) $h$ only and for $n=2 ; p=0,0.5,1$;

(c) $p$ only and for $n=1,2,3 ; h=0.25$

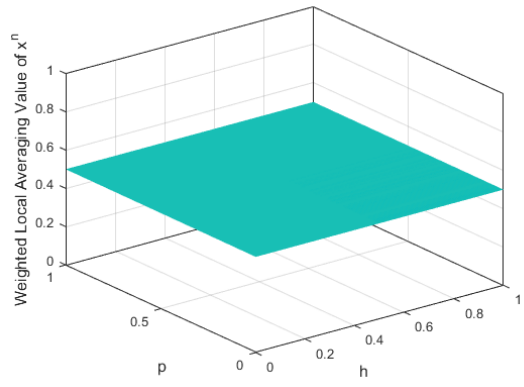

(a)

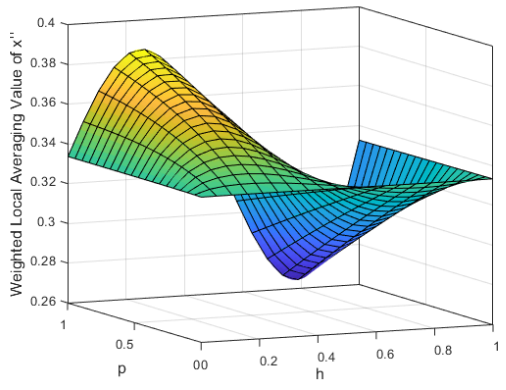

(b)

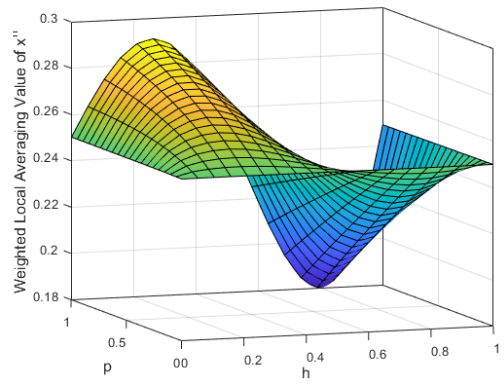

(c)

Fig. 2. WLA value of $x^{n}$ as a function of $h$ and $p$ with (a) $n=1$; (b) $n=2$ and (c) $n=3$

the weighted local average of $g(x)$ can be formulated in its explicit form using the linearity of the weighted averaging operator and Eq. (8)

$$
\langle g(x), h\rangle_{p}=\left\langle\sum_{i=0}^{\infty} g_{i} x^{i}, h\right\rangle_{p}=\sum_{i=0}^{\infty} g_{i}\left\langle x^{i}, h\right\rangle_{p}=\sum_{i=0}^{\infty} \frac{g_{i}}{i+1}\left(1+p\left(1-2 h+h^{i+1}-(1-h)^{i+1}\right)\right) .
$$

The determination of weights for a data given is a difficult and complicated problem which needs an adequate strategy. So far there is no general theory about this. In this paper, we consider three weight values that are $p=0.25,0.5$ and 1 . To clarify the meaning of the parameter of boundary regulation one represents Eq. (6) in the following form

$$
\begin{aligned}
\langle g(x), h\rangle_{p} & =(1-2 p h) \int_{0}^{1} g(x) \mathrm{d} x+p\left(\int_{0}^{h} g(x) \mathrm{d} x+\int_{1-h}^{1} g(x) \mathrm{d} x\right) \\
& =\int_{0}^{1} g(x) \mathrm{d} x+p\left(\int_{0}^{h} g(x) \mathrm{d} x+\int_{1-h}^{1} g(x) \mathrm{d} x-2 h \int_{0}^{1} g(x) \mathrm{d} x\right) .
\end{aligned}
$$

The expression

$$
B(g(x), h)=\left(\int_{0}^{h} g(x) \mathrm{d} x+\int_{1-h}^{1} g(x) \mathrm{d} x-2 h \int_{0}^{1} g(x) \mathrm{d} x\right),
$$

can be interpreted as a measure charactering the effect of boundary values of the function $g(x)$. It is seen from Eq. (13) when $p=0.5$ the influence level of the effect of boundary values is equal to a half of the one of the averaged value of the function $g(x)$, and when $p=1$ two influence levels are considered 
the same. A detailed investigation of $B(g(x), h)$ might be an interesting topic of coming research. In the next sections two applications of WLA will be illustrated for the buckling problem of Euler columns and the frequency analysis of free vibration of strongly nonlinear systems.

\section{APPLICATION OF WLA TO THE BUCKLING PROBLEM OF EULER COLUMNS}

For the elastic buckling of Euler columns [11] consider an elastic column of length $L$ subjected to an axial compressive force $\bar{P}$. The column undergoes a lateral deflection denoted by $\bar{W}(z)$, where $z$ denotes the vertical axis coordinate of the column. Using the theory of Euler-Bernoulli beam the lateral deflection of the column is described by the following differential equation

$$
\frac{\mathrm{d}^{2}}{\mathrm{~d} z^{2}}\left(E I(z) \frac{\mathrm{d}^{2} \bar{W}(z)}{\mathrm{d} z^{2}}\right)+\bar{P} \frac{\mathrm{d}^{2} \bar{W}(z)}{\mathrm{d} z^{2}}=0,
$$

where is the Young's modulus of elasticity, $I(z)$ is the moment of inertia of the column. Let $I(z)=I_{0} b(z)$, where $I_{0}$ is the moment of inertia of the column at $z=0$ and $b(z)$ is a function defined in the interval $[0, L]$ and $b(z)>0, \forall z \in[0, L]$. By using transformation

$$
x=z / L, \quad W(x)=\bar{W}(z) / L, \quad P=\bar{P} L^{2} / E I_{0},
$$

one gets from Eq. (15)

$$
\frac{\mathrm{d}^{2}}{\mathrm{~d} x^{2}}\left(b(x) \frac{\mathrm{d}^{2} W(x)}{\mathrm{d} x^{2}}\right)+P \frac{\mathrm{d}^{2} W(x)}{\mathrm{d} x^{2}}=0 .
$$

To solve Eq. (16) one needs to add boundary conditions for column at two points $x=0$ and $x=1$. In this paper we consider 4 typical types of boundary conditions as given in Tab. 1. For each type of boundary conditions there exists a corresponding comparison function $W(x)$ of polynomial form, $W(x)=\sum_{i=0}^{n=4} C_{i} x^{i}$, where $C_{i}$ are obtained from the boundary conditions. Tab. 1 shows 4 types of boundary conditions and corresponding comparison functions.

\begin{tabular}{|c|c|c|}
\hline Type of boundary conditions & Boundary conditions & Comparison function $W(x)$ \\
\hline Pinned-Pinned Column (P-P) & $\begin{array}{l}W(0)=0 ; \frac{\mathrm{d}^{2} W(0)}{\mathrm{d} x^{2}}=0 \\
W(1)=0 ; \frac{\mathrm{d}^{2} W(1)}{\mathrm{d} x^{2}}=0\end{array}$ & $x^{4}-2 x^{3}+x$ \\
\hline Clamped-Pinned Column (C-P) & $\begin{array}{l}W(0)=0 ; \frac{\mathrm{d} W(0)}{\mathrm{d} x}=0 \\
W(1)=0 ; \frac{\mathrm{d}^{2} W(1)}{\mathrm{d} x^{2}}=0\end{array}$ & $2 x^{4}-5 x^{3}+3 x^{2}$ \\
\hline Clamped-Sliding Column (C-S) & $\begin{array}{c}W(0)=0 ; \frac{\mathrm{d} W(0)}{\mathrm{d} x}=0 \\
\frac{\mathrm{d} W(1)}{\mathrm{d} x}=0 ; \frac{\mathrm{d}^{3} W(1)}{\mathrm{d} x^{3}}+P \frac{\mathrm{d} W(1)}{\mathrm{d} x}=0\end{array}$ & $x^{4}-4 x^{3}+4 x^{2}$ \\
\hline Clamped-Clamped Column (C-C) & $\begin{array}{l}W(0)=0 ; \frac{\mathrm{d} W(0)}{\mathrm{d} x}=0 \\
W(1)=0 ; \frac{\mathrm{d} W(1)}{\mathrm{d} x}=0\end{array}$ & $x^{4}-2 x^{3}+x^{2}$ \\
\hline
\end{tabular}

Table 1. Different types of columns with corresponding boundary conditions and comparison functions

The first order approximate solution of the buckling problem described by Eq. (16) can be obtained by using Galerkin method for one term comparison function as follows

$$
\left\langle\left(\frac{\mathrm{d}^{2}}{\mathrm{~d} x^{2}}\left(b(x) \frac{\mathrm{d}^{2} W(x)}{\mathrm{d} x^{2}}\right)+P \frac{\mathrm{d}^{2} W(x)}{\mathrm{d} x^{2}}\right) W(x)\right\rangle=0 .
$$


Eq. (17) leads to the approximate buckling load obtained by Galerkin method with CA

$$
P_{c a}=-\frac{\left\langle\frac{\mathrm{d}^{2}}{\mathrm{~d} x^{2}}\left(b(x) \frac{\mathrm{d}^{2} W(x)}{\mathrm{d} x^{2}}\right) W(x)\right\rangle}{\left\langle\frac{\mathrm{d}^{2} W(x)}{\mathrm{d} x^{2}} W(x)\right\rangle} .
$$

If in Eq. (18) one replaces the conventional averaging by the weighted local averaging and gets

$$
\left\langle\left(\frac{\mathrm{d}^{2}}{\mathrm{~d} x^{2}}\left(b(x) \frac{\mathrm{d}^{2} W(x)}{\mathrm{d} x^{2}}\right)+P \frac{\mathrm{d}^{2} W(x)}{\mathrm{d} x^{2}}\right) W(x), h\right\rangle_{p}=0 .
$$

Then one has the approximate buckling load obtained by Galerkin method with WLA

$$
P(p, h)=-\frac{\left\langle\frac{\mathrm{d}^{2}}{\mathrm{~d} x^{2}}\left(b(x) \frac{\mathrm{d}^{2} W(x)}{\mathrm{d} x^{2}}\right) W(x), h\right\rangle_{p}}{\left\langle\frac{\mathrm{d}^{2} W(x)}{\mathrm{d} x^{2}} W(x), h\right\rangle_{p}} .
$$

Or in the explicit form

$$
\begin{aligned}
& P(p, h)= \\
& -\frac{(1-2 p h) \int_{0}^{1} \frac{\mathrm{d}^{2}}{\mathrm{~d} x^{2}}\left(b(x) \frac{\mathrm{d}^{2} W(x)}{\mathrm{d} x^{2}}\right) W(x) \mathrm{d} x+p\left(\int_{0}^{h} \frac{\mathrm{d}^{2}}{\mathrm{~d} x^{2}}\left(b(x) \frac{\mathrm{d}^{2} W(x)}{\mathrm{d} x^{2}}\right) W(x) \mathrm{d} x+\int_{1-h}^{1} \frac{\mathrm{d}^{2}}{\mathrm{~d} x^{2}}\left(b(x) \frac{\mathrm{d}^{2} W(x)}{\mathrm{d} x^{2}}\right) W(x) \mathrm{d} x\right)}{(1-2 p h) \int_{0}^{1} \frac{\mathrm{d}^{2} W(x)}{\mathrm{d} x^{2}} W(x) \mathrm{d} x+p\left(\int_{0}^{h} \frac{\mathrm{d}^{2} W(x)}{\mathrm{d} x^{2}} W(x) \mathrm{d} x+\int_{1-h}^{1} \frac{\mathrm{d}^{2} W(x)}{\mathrm{d} x^{2}} W(x) \mathrm{d} x\right)} .
\end{aligned}
$$

It is seen from Eq. (21) that the critical load determined by Galerkin method with WLA is a function of parameter $p$ and local value $h$. If the value $p$ is given, the buckling load will be chosen as the lowest value of $P(p, h)$ in the interval [0.1] i.e.

$$
P_{\text {wla }}(p)=\min _{h \in[0,1]} P(p, h) .
$$

It is clearly seen from Eqs. (18) and (21) that the approximate buckling load obtained by Galerkin method with CA is corresponding to the case $p=0$ of the approximate buckling load obtained by Galerkin method with WLA.

\subsection{Columns with constant cross-section}

Tab. 2 shows the buckling loads obtained by Galerkin method with CA $(p=0)$ and WLA $(p=0.25$ and $p=0.5$ ) for all 4 types of boundary conditions and compares those with exact values from [8].

Table 2. Accuracy of approximate buckling loads for different types of column with constant cross-section

\begin{tabular}{crcccccc}
\hline Type of $B C$ & $P_{\text {exact }}[8]$ & $P_{c a}=P_{\text {wla }}, p=0$ & $\% E$ & $P_{\text {wla }}, p=0.25$ & $\% E$ & $P_{\text {wla }}, p=0.5$ & $\% E$ \\
\hline P-P & 9.8696 & 9.8823 & 0.129 & 9.6474 & 2.252 & 9.443 & 4.326 \\
C-P & 20.1907 & 21.0000 & 4.008 & 20.3582 & 0.833 & 19.8113 & 1.876 \\
C-S & 9.8696 & 10.5000 & 6.387 & 9.9931 & 1.252 & 9.5289 & 3.452 \\
C-C & 39.4784 & 42.0000 & 6.387 & 38.9438 & 1.358 & 36.591 & 7.319 \\
\hline
\end{tabular}

It is seen from Tab. 2 that percent errors of solutions obtained by WLA for $p=0.25$ are much smaller than the ones of solutions obtained by of CA for 3 types of columns (C-P, C-S, C-C), for example, for C-P type two errors are $4.008 \%$ and $0.833 \%$, respectively Besides, the accuracy of WLA for $p=0.25$ is much better in average compared to the accuracy of CA. The percent errors of WLA for $p=0.5$ are smaller than the ones of CA for 2 types of columns (C-P and C-S) and larger than the ones of CA for 2 
remaining types of columns (P-P and C-C). The accuracy of WLA for $p=0.5$ and of CA is nearly the same in average.

\subsection{Columns with variable cross-section given by exponential function}

In this section, we consider columns with variable cross-section whose moment of inertia is given by exponential function

$$
E I(x)=E I_{0} \exp (-B L x) .
$$

Tabs. 3-6 show the critical buckling loads obtained by WLA for $p=0,25$ and $p=0.5$ as well as the ones of CA for 4 types of columns with variable cross-section. It is seen from all tables that the accuracy of solutions obtained by WLA is significantly improved in comparison to the one of solutions obtained by of CA, specially, when the change of the cross-section is increased. For example, in Tab. 3 starting from $B L=1,2$ the percent errors of WLA for $p=0.25$ are $0.728 \%$ and $10.079 \%$ while the ones of CA are $4.714 \%$ and $19.560 \%$, respectively. The accuracy of solutions obtained by WLA for $p=0.5$ is much improved, namely for $B L=1.5$ and 2 the percent errors of WLA are $0.806 \%$ and $1.821 \%$ while the ones of solutions obtained by CA are $10.698 \%$ and $19.560 \%$, respectively. In Tabs. $4-6$ the accuracy of solutions obtained by WLA is always much better than the one of solutions obtained by CA when $B L>0$. For columns with variable cross-section the accuracy of solutions obtained by WLA for $p=0.5$ is much better than the accuracy of solutions obtained by WLA for $p=0.25$.

Table 3. Accuracy of approximate buckling loads for Pinned-Pinned column with exponential moment of inertia

\begin{tabular}{lccccccc}
\hline \multicolumn{1}{c}{$B L$} & $P_{\text {exact }}[8]$ & $P_{c a}=P_{\text {wla }}, p=0$ & $\% E$ & $P_{\text {wla }}, p=0.25$ & $\% E$ & $P_{\text {wla }}, p=0.5$ & $\% E$ \\
\hline 0 & 9.8696 & 9.8823 & 0.129 & 9.6474 & 2.252 & 9.4427 & 4.326 \\
0.5 & 7.6340 & 7.7308 & 1.268 & 7.5192 & 1.504 & 7.3348 & 3.920 \\
1 & 5.8270 & 6.1017 & 4.714 & 5.869 & 0.728 & 5.6671 & 2.745 \\
1.5 & 4.3890 & 4.8585 & 10.698 & 4.589 & 4.551 & 4.3536 & 0.806 \\
2 & 3.2640 & 3.9024 & 19.560 & 3.593 & 10.079 & 3.3234 & 1.821 \\
\hline
\end{tabular}

Table 4. Accuracy of approximate buckling loads for Clamped-Pinned column with exponential moment of inertia

\begin{tabular}{lrcrcrrr}
\hline \multicolumn{1}{c}{$B L$} & $P_{\text {exact }}[8]$ & $P_{c a}=P_{\text {wla }}, p=0$ & $\% E$ & $P_{\text {wla }}, p=0.25$ & $\% E$ & $P_{\text {wla }}, p=0.5$ & $\% E$ \\
\hline 0 & 20.1907 & 21.0000 & 4.008 & 20.3582 & 0.833 & 19.8113 & 1.876 \\
0.5 & 15.6400 & 17.2408 & 10.236 & 16.8155 & 7.516 & 16.4524 & 5.194 \\
1 & 11.9900 & 14.4716 & 20.698 & 14.1140 & 17.714 & 13.8081 & 15.164 \\
1.5 & 9.0980 & 12.4117 & 36.422 & 12.0412 & 32.350 & 11.7241 & 28.865 \\
2 & 6.8390 & 10.8627 & 58.835 & 10.4401 & 52.656 & 10.0786 & 47.369 \\
\hline
\end{tabular}

Table 5. Accuracy of approximate buckling loads for Clamped-Sliding column with exponential moment of inertia

\begin{tabular}{lccccccc}
\hline \multicolumn{1}{c}{$B L$} & $P_{\text {exact }}[8]$ & $P_{c a}=P_{\text {wla }}, p=0$ & $\% E$ & $P_{\text {wla }}, p=0.25$ & $\% E$ & $P_{\text {wla }}, p=0.5$ & $\% E$ \\
\hline 0 & 9.8696 & 10.5000 & 6.387 & 9.9931 & 1.252 & 9.5289 & 3.452 \\
0.5 & 7.6830 & 9.8408 & 28.086 & 9.1585 & 19.205 & 8.5336 & 11.071 \\
1 & 5.9730 & 8.8815 & 48.694 & 8.1329 & 36.161 & 7.4473 & 24.682 \\
1.5 & 4.6330 & 7.9233 & 71.019 & 7.1769 & 54.907 & 6.4931 & 40.150 \\
2 & 3.5800 & 7.0808 & 97.788 & 6.3760 & 78.100 & 5.7304 & 60.066 \\
\hline
\end{tabular}

Table 6. Accuracy of approximate buckling loads for Clamped-Clamped column with exponential moment of inertia

\begin{tabular}{lccccccc}
\hline BL & $P_{\text {exact }}[8]$ & $P_{c a}=P_{\text {wla }}, p=0$ & $\% E$ & $P_{\text {wla }}, p=0.25$ & $\% E$ & $P_{\text {wla }}, p=0.5$ & $\% E$ \\
\hline 0 & 39.4784 & 42.0000 & 6.387 & 38.9438 & 1.358 & 36.5906 & 7.319 \\
0.5 & 30.6000 & 33.2473 & 8.651 & 30.7427 & 0.4663 & 28.8142 & 5.836 \\
1 & 23.4900 & 27.1709 & 15.670 & 24.7742 & 6.108 & 23.1952 & 1.255 \\
1.5 & 17.8600 & 22.8761 & 28.086 & 20.3307 & 16.072 & 19.0786 & 6.823 \\
2 & 13.4600 & 19.7815 & 46.965 & 17.1182 & 31.183 & 16.0222 & 19.036 \\
\hline
\end{tabular}




\section{APPLICATION OF WLA TO THE FREQUENCY ANALYSIS OF STRONGLY NONLINEAR SYSTEMS}

In this section the second application of the weighted local averaging is one to the analysis of free vibration of strongly nonlinear systems which have attracted an increasing interest of scientists and engineers in the field of dynamic nonlinear problems [12-19]. For strongly nonlinear systems there exists no small parameter in the governing equation so the traditional perturbation methods cannot be applied directly. Many techniques have appeared in the literature for strongly nonlinear systems, for example, the weighted linearization method [12], the modified Lindstedt-Poincare method [13], the perturbation incremental method [14], equivalent non-linearization method [15] and the homotopy perturbation method [16].

We consider the free vibration of a strongly nonlinear system described by the following differential equation

$$
\ddot{z}(t)+f(z(t))=0, \quad z(0)=A, \quad \dot{z}(0)=0,
$$

where dot denotes the differentiation with respect to $t, f(z)$ is a nonlinear function of $z, A$ is a constant value. We will investigate the frequency dependence on the initial amplitude $A$ of a periodic solution of Eq. (24) by Galerkin method with WLA. To do that we seek the approximate periodic solution of Eq. (24) in the form

$$
z(t)=A \cos \omega t=\cos 2 \pi x,
$$

where $\omega$ is unknown frequency and $x$ is a new variable which is related to the variable $t$ as follows

$$
x=\frac{\omega}{2 \pi} t
$$

It is seen from Eq. (26) that in a period of vibration, $T$, one has

$$
0 \leq t \leq T=\frac{2 \pi}{\omega} \text { then } 0 \leq x \leq 1 .
$$

Substituting Eq. (25) into Eq. (24) one gets the residual of equation

$$
R(t)=\ddot{z}(t)+f(z(t))=-\omega^{2} A \cos \omega t+f(A \cos \omega t),
$$

or

$$
R(x)=-\omega^{2} A \cos \omega t+f(A \cos \omega t)=-\omega^{2} A \cos 2 \pi x+f(A \cos 2 \pi x) .
$$

Using Galerkin method with CA one gets the condition of orthogonality in the form

$$
\begin{aligned}
\frac{\omega}{2 \pi} \int_{0}^{2 \pi / \omega} R(t) A \cos \omega t \mathrm{~d} t & =\int_{0}^{1} R(x) A \cos 2 \pi x \mathrm{~d} x \\
& =\int_{0}^{1}\left(-\omega^{2} A \cos 2 \pi x+f(A \cos 2 \pi x)\right) A \cos 2 \pi x \mathrm{~d} x=0 .
\end{aligned}
$$

Hence the approximate frequency $\omega_{c a}$ obtained by Galerkin method with CA takes the expression

$$
\omega_{c a}^{2}=\frac{\int_{0}^{1} f(A \cos 2 \pi x) \cos 2 \pi x \mathrm{~d} x}{A \int_{0}^{1} \cos ^{2} 2 \pi x \mathrm{~d} x} .
$$

Using Galerkin method with WLA one writes the condition of orthogonality in the form

$$
\left\langle\left(-\omega^{2} A \cos 2 \pi x+f(A \cos 2 \pi x)\right) A \cos 2 \pi x, h\right\rangle_{p}=0,
$$


and the approximate frequency $\omega$ obtained by Galerkin method with WLA takes the expression

$$
\frac{(1-2 p h) \int_{0}^{1} f(A \cos 2 \pi x) \cos 2 \pi x \mathrm{~d} x+p\left(\int_{0}^{h} f(A \cos 2 \pi x) \cos 2 \pi x \mathrm{~d} x+\int_{1-h}^{1} f(A \cos 2 \pi x) \cos 2 \pi x \mathrm{~d} x\right)}{(1-2 p h) \int_{0}^{1} A \cos ^{2} 2 \pi x \mathrm{~d} x+p\left(\int_{0}^{h} A \cos ^{2} 2 \pi x \mathrm{~d} x+\int_{1-h}^{1} A \cos ^{2} 2 \pi x \mathrm{~d} x\right)} .
$$

It is seen from Eq. (33) that the frequency determined by Galerkin method with WLA is a function of parameter $p$ and local value $h$. When the value $p$ is given, the frequency interested, $\omega_{w l a}$, will be chosen as the lowest value of $\omega(p, h)$ in the interval (0.1) i.e.

$$
\omega_{\text {wla }}(p)=\min _{h \in[0,1]} \omega(p, h) .
$$

It is seen that the approximate frequency $\omega_{c a}$ obtained by CA (Eq. (31)) can be obtained from the approximate frequency $\omega_{\text {wla }}$ obtained by WLA (Eq. (33)) by putting $p=0$.

In the next subsections some typical strongly nonlinear systems are considered and the accuracy of approximate frequencies obtained by WLA and CA are evaluated, respectively.

\subsection{Generalized Duffing system with strong nonlinearity}

A generalized Duffing system with strong nonlinearity has the form

$$
\ddot{z}(t)+z^{2 n+1}(t)=0, \quad z(0)=A, \quad \dot{z}(0)=0,
$$

which is obtained from Eq. (24) when the nonlinear function $f(z)$ takes the form

$$
f(z)=z^{2 n+1},
$$

where $n$ is a positive, integer number. Substituting Eq. (36) into Eq. (33) leads to the following approximate frequency for the generalized Duffing system (35)

$$
\omega_{\text {wla }}^{2}=\frac{(1-2 p h) \int_{0}^{1} A^{2 n} \cos ^{2 n+2} 2 \pi x \mathrm{~d} x+p\left(\int_{0}^{h} A^{2 n} \cos ^{2 n+2} 2 \pi x \mathrm{~d} x+\int_{1-h}^{1} A^{2 n} \cos ^{2 n+2} 2 \pi x \mathrm{~d} x\right)}{(1-2 p h) \int_{0}^{1} \cos ^{2} 2 \pi x \mathrm{~d} x+p\left(\int_{0}^{h} \cos ^{2} 2 \pi x \mathrm{~d} x+\int_{1-h}^{1} \cos ^{2} 2 \pi x \mathrm{~d} x\right)} .
$$

Zhang [20] obtains the following approximate natural frequency for Eq. (35) using He's energy balance method.

$$
\omega_{E B M}=\sqrt{\frac{3 A^{2 n}}{n+1}\left(1-\frac{(2 n+2) ! !}{(2 n+3) ! !}\right)} .
$$

The exact frequency is determined by the following formula [20]

$$
\omega_{E}=\frac{2 \pi}{4 \int_{0}^{\pi / 2} \frac{\mathrm{d} \theta}{\sqrt{Q}}}, \quad Q=\sqrt{\frac{1}{n+1} A^{2 n}\left(1+\sin ^{2} \theta+\sin ^{4} \theta+\ldots+\sin ^{2 n} \theta\right)} .
$$

Tab. 7 shows the approximate frequencies obtained by He's energy balance method (Eq. (38)) and Galerkin method with CA and WLA (Eq. (37)) for different values of integer $n$ and compares those with exact frequency (Eq. (39)).

It is seen from Tab. 7 that the accuracy of solutions obtained by WLA for all values $p=0.25 ; 0.5 ; 1$ is always better than the accuracy of solutions obtained by CA, specially, when $n$ is increasing. For example, for $n=1 ; 2$ the percent errors of WLA for $p=0.25 ; 0.5 ; 1$ are $1.313 \%, 4.324 \% ; 0.311 \%, 2.653 \%$; $1.958 \%, 1.168 \%$ while the ones of CA are $2.224 \%, 5.864 \%$, respectively; for $n=4,5$ the percent errors of 
Table 7. Comparison of approximate frequencies with exact frequency

\begin{tabular}{|c|c|c|c|c|c|c|c|c|c|c|c|}
\hline$n$ & $\begin{array}{c}\omega_{E} / A^{n} \\
{[20]}\end{array}$ & $\begin{array}{c}\omega_{c a} / A^{n}= \\
\omega_{w l a} / A^{n}, \\
p=0\end{array}$ & $E \%$ & $\begin{array}{c}\omega_{\text {wla }} / A^{n} \\
p=0.25\end{array}$ & $E \%$ & $\begin{array}{c}\omega_{\text {wla }} / A^{n} \\
p=0.5\end{array}$ & $E \%$ & $\begin{array}{c}\omega_{\text {wla }} / A^{n} \\
p=1\end{array}$ & $E \%$ & $\begin{array}{c}\omega_{E B M} / A^{n}, \\
{[20]}\end{array}$ & $E \%$ \\
\hline 1 & 0.8472 & 0.8660 & 2.224 & 0.8583 & 1.313 & 0.8498 & 0.311 & 0.8306 & 1.958 & 0.8367 & 1.25 \\
\hline 2 & 0.7468 & 0.7906 & 5.864 & 0.7791 & 4.324 & 0.7667 & 2.653 & 0.7381 & 1.168 & 0.7368 & 1.34 \\
\hline 3 & 0.6750 & 0.7395 & 9.559 & 0.7259 & 7.543 & 0.7111 & 5.347 & 0.6771 & 0.308 & 0.6673 & 1.14 \\
\hline 4 & 0.6204 & 0.7016 & 13.082 & 0.6865 & 10.665 & 0.6702 & 8.026 & 0.6326 & 1.972 & 0.6151 & 0.85 \\
\hline 5 & 0.5772 & 0.6717 & 16.376 & 0.6557 & 13.615 & 0.6383 & 10.594 & 0.5983 & 3.660 & 0.5740 & 0.54 \\
\hline
\end{tabular}

WLA for $p=0.25 ; 0.5 ; 1$ are $10.665 \%, 13.615 \% ; 8.026 \%, 10.594 \% ; 1.972 \%, 3.660 \%$ while the ones of CA are $13.082 \%, 16.376 \%$, respectively. The percent errors of WLA for $p=1$ are smaller than the ones of He's energy balance method for $n=2$ and 3; while they are bigger for $n=1,4$ and 5 .

\subsection{Nonlinear system with discontinuity term}

The second illustration example is the nonlinear oscillator with discontinuity term for which the elastic restoring force is an antisymmetric constant force

$$
f(z)=\operatorname{sgn}(z)
$$

where the function $\operatorname{sgn}(z)$ is defined by

$$
\operatorname{sgn}(z)=\left\{\begin{array}{cc}
-1, & z<0 \\
0, & z=0 \\
1, & z>0
\end{array}\right.
$$

Substituting Eq. (40) into Eq. (24) leads to the following equation

$$
\ddot{z}(t)+\operatorname{sgn}(z(t))=0, \quad z(0)=A, \quad \dot{z}(0)=0 .
$$

This type of nonlinear system has been analyzed by many authors using different analytical approximate methods [21-24]. The exact frequency of the nonlinear system (42) is given in [25]

$$
\omega_{E}=\pi /(2 \sqrt{2 A})=1.110721 / \sqrt{A} .
$$

Substituting Eq. (40) into Eq. (33) leads to the following approximate frequency for the nonlinear system with discontinuity term (42)

$$
\begin{aligned}
& \omega_{\text {wla }}^{2}= \\
& \frac{(1-2 p h) \int_{0}^{1} A \operatorname{sgn}(\cos 2 \pi x) \cos 2 \pi x \mathrm{~d} x+p\left(\int_{0}^{h} A \operatorname{sgn}(\cos 2 \pi x) \cos 2 \pi x \mathrm{~d} x+\int_{1-h}^{1} A \operatorname{sgn}(\cos 2 \pi x) \cos 2 \pi x \mathrm{~d} x\right)}{(1-2 p h) \int_{0}^{1} A^{2} \cos ^{2} 2 \pi x \mathrm{~d} x+p\left(\int_{0}^{h} A^{2} \cos ^{2} 2 \pi x \mathrm{~d} x+\int_{1-h}^{1} A^{2} \cos ^{2} 2 \pi x \mathrm{~d} x\right)} .
\end{aligned}
$$

Tab. 8 shows the approximate frequency obtained by He's energy balance method [20] and Galerkin method with CA and WLA (Eq. (44)) for different values of $p$ and compares those with exact frequency [25].

Table 8. Comparison of approximate frequencies with exact frequency

\begin{tabular}{ccccccccccc}
\hline $\begin{array}{c}\sqrt{A} \omega_{E} \\
{[25]}\end{array}$ & $\begin{array}{c}\sqrt{A} \omega_{\text {ca }}= \\
\sqrt{A} \omega_{\text {wla }} \\
p=0\end{array}$ & $E \%$ & $\begin{array}{c}\sqrt{A} \omega_{\text {wlal }} \\
p=0.25\end{array}$ & $E \%$ & $\begin{array}{c}\sqrt{A} \omega_{\text {wlal }} \\
p=0.5\end{array}$ & $E \%$ & $\begin{array}{c}\sqrt{A} \omega_{\text {wlal }} \\
p=1\end{array}$ & $E \%$ & $\begin{array}{c}\sqrt{A} \omega_{E B M} \\
{[20]}\end{array}$ & $E \%$ \\
\hline 1.1107 & 1.1284 & 1.594 & 1.1205 & 0.882 & 1.1132 & 0.225 & 1.0998 & 0.981 & 1.1347 & 2.161 \\
\hline
\end{tabular}


It is seen from Tab. 8 that the accuracy of solutions obtained by WLA for all values $p=0.25 ; 0.5 ; 1$ is better than the accuracies of solutions obtained by CA and by He's energy balance method; and the accuracy of WLA for $p=0.5$ is the best.

\subsection{System with rational elastic restoring term}

Consider the strongly nonlinear oscillator of the form

$$
\ddot{z}(t)+1 / z(t)=0, \quad z(0)=A, \quad \dot{z}(0)=0 .
$$

In this case the nonlinear function is of follows

$$
f(z)=1 / z \text {. }
$$

The approximate frequency obtained by He [16] using the homotopy perturbation method has the expression

$$
\omega_{h p m}=1.1547 / A \text {. }
$$

The exact frequency of the system (45) is given in [25]

$$
\omega_{E}=\frac{\sqrt{2 \pi}}{2 A}=1.2533 / A .
$$

Substituting Eq. (46) into Eq. (33) leads to the following approximate frequency for the system (45)

$$
\begin{aligned}
& \omega_{w l a}^{2}=\frac{(1-2 p h) \int_{0}^{1} 1+p\left(\int_{0}^{h} 1 \mathrm{~d} x+\int_{1-h}^{1} 1 \mathrm{~d} x\right)}{(1-2 p h) \int_{0}^{1} A^{2} \cos ^{2} 2 \pi x \mathrm{~d} x+p\left(\int_{0}^{h} A^{2} \cos ^{2} 2 \pi x \mathrm{~d} x+\int_{1-h}^{1} A^{2} \cos ^{2} 2 \pi x \mathrm{~d} x\right)} \\
& =\frac{1}{A^{2}\left[(1-2 p h) \int_{0}^{1} \cos ^{2} 2 \pi x \mathrm{~d} x+p\left(\int_{0}^{h} \cos ^{2} 2 \pi x \mathrm{~d} x+\int_{1-h}^{1} \cos ^{2} 2 \pi x \mathrm{~d} x\right)\right]} \text {. }
\end{aligned}
$$

Tab. 9 shows the approximate frequencies obtained by the homotopy perturbation method (Eq. (47)) and Galerkin method with CA and WLA (Eq. (49)), and compares those with exact frequency (Eq. (48)).

Table 9. Comparison of approximate frequencies with exact frequency

\begin{tabular}{ccccccccccc}
\hline $\begin{array}{c}A \omega_{E} \\
{[25]}\end{array}$ & $\begin{array}{c}A \omega_{\text {wlal }} \\
p=0\end{array}$ & $\begin{array}{c}\text { error } \\
\%\end{array}$ & $\begin{array}{c}A \omega_{\text {wlal }} \\
p=0.25\end{array}$ & $\begin{array}{c}\text { error } \\
\%\end{array}$ & $\begin{array}{c}A \omega_{\text {wla }} \\
p=0.5\end{array}$ & $\begin{array}{c}\text { error } \\
\%\end{array}$ & $\begin{array}{c}A \omega_{\text {wlal }} \\
p=1\end{array}$ & $\begin{array}{c}\text { error } \\
\%\end{array}$ & $\begin{array}{c}A \omega_{\text {hpm }} \\
{[16]}\end{array}$ & $\begin{array}{c}\text { error } \\
\%\end{array}$ \\
\hline 1.2533 & 1.4142 & 12.839 & 1.3869 & 10.659 & 1.3611 & 8.601 & 1.3135 & 4.803 & 1.1547 & 7.867 \\
\hline
\end{tabular}

It is seen from Tab. 9 that the accuracy of solution obtained by WLA for all values $p=0.25 ; 0.5 ; 1$ is better than the accuracy of solution obtained by CA. The percent error of solution obtained by WLA for $p=1$ is smaller than the one of solution obtained by the homotopy perturbation method, however, the percent errors of solution obtained by WLA for $p=0.25$ and $p=0.5$ are bigger than the one of solution obtained by the homotopy perturbation method.

\subsection{Duffing-harmonic oscillator}

Consider a free oscillator with a rational form of the restoring force which is governed by the following equation

$$
\ddot{z}+\frac{z^{3}}{1+z^{2}}=0, \quad z(0)=A, \quad \dot{z}(0)=0 .
$$

In this case the nonlinear function takes the form

$$
f(z)=\frac{z^{3}}{1+z^{2}}
$$


It is interesting to note that for small $z$, Eq. (50) is closed to the Duffing nonlinear oscillator, while for the large $z$, the equation approximates a linear oscillator; For this reason the system (50) is called the Duffing-harmonic oscillator.

The investigation of this nonlinear oscillator was started by Mickens [26] and then continued by many authors [27-32]. Substituting Eq. (51) into Eq. (33) leads to the following approximate frequency for the Duffing-harmonic oscillator (50)

$$
\begin{aligned}
& \omega_{\text {wla }}^{2}= \\
& \frac{(1-2 p h) \int_{0}^{1} \frac{(A \cos 2 \pi x)^{3}}{1+(A \cos 2 \pi x)^{2}} \cos 2 \pi x \mathrm{~d} x+p\left(\int_{0}^{h} \frac{(A \cos 2 \pi x)^{3}}{1+(A \cos 2 \pi x)^{2}} \cos 2 \pi x \mathrm{~d} x+\int_{1-h}^{1} \frac{(A \cos 2 \pi x)^{3}}{1+(A \cos 2 \pi x)^{2}} \cos 2 \pi x \mathrm{~d} x\right)}{(1-2 p h) \int_{0}^{1} A \cos ^{2} 2 \pi x \mathrm{~d} x+p\left(\int_{0}^{h} A \cos ^{2} 2 \pi x \mathrm{~d} x+\int_{1-h}^{1} A \cos ^{2} 2 \pi x \mathrm{~d} x\right)} .
\end{aligned}
$$

The exact frequency is presented in [29] as follows

$$
\omega_{E}=\frac{\pi}{2}\left(\int_{0}^{\pi / 2} \frac{A \cos t \mathrm{~d} t}{\sqrt{A^{2} \cos ^{2} t+\ln \left[1-A^{2} \cos ^{2} t /\left(1+A^{2}\right)\right]}}\right)^{-1} .
$$

The approximate frequency for the system (50) obtained by the parameter-expansion method (PEM) is shown in [33]

$$
\omega_{P E M}=\sqrt{\frac{3 A^{2}}{4+3 A^{2}}}
$$

Table 10. Comparison of approximate frequencies with exact frequency

\begin{tabular}{cccccccccccc}
\hline \multirow{2}{*}{$A$} & $\begin{array}{c}\omega_{E} \\
{[29]}\end{array}$ & $\begin{array}{c}\omega_{\text {wla }} \\
p=0\end{array}$ & $\begin{array}{c}\text { error } \\
\%\end{array}$ & $\begin{array}{c}\omega_{\text {wla }} \\
p=0.25\end{array}$ & $\begin{array}{c}\text { error } \\
\%\end{array}$ & $\begin{array}{c}\omega_{\text {wla, }} \\
p=0.5\end{array}$ & $\begin{array}{c}\text { error } \\
\%\end{array}$ & $\begin{array}{c}\omega_{\text {wla }} \\
p=1\end{array}$ & $\begin{array}{c}\text { error } \\
\%\end{array}$ & $\begin{array}{c}\omega_{P E M} \\
{[33]}\end{array}$ & $\begin{array}{c}\text { error } \\
\%\end{array}$ \\
\hline 0.1 & 0.08349 & 0.08624 & 3.299 & 0.08548 & 2.377 & 0.08464 & 1.378 & 0.08274 & 0.903 & 0.08628 & 3.342 \\
0.5 & 0.38737 & 0.39423 & 1.771 & 0.39108 & 0.957 & 0.38766 & 0.074 & 0.37990 & 1.942 & 0.39736 & 2.579 \\
1 & 0.63678 & 0.64359 & 1.070 & 0.63962 & 0.445 & 0.63530 & 0.233 & 0.62543 & 1.783 & 0.65465 & 2.807 \\
\hline
\end{tabular}

Tab. 10 shows the approximate frequencies obtained by PEM (Eq. (54)) and Galerkin method with CA and WLA (Eq. (52)), and compares those with exact frequency (Eq. (53)). It is seen from Tab. 10 that the accuracy of solution obtained by WLA for $p=0.25 ; 0.5$ is better than the accuracy of solution obtained by CA. The percent errors of solution obtained by WLA for all values $p=0.25 ; 0.5 ; 1$ are smaller than the ones of solution obtained by the parameter-expansion method.

\section{CONCLUSIONS}

The averaged values play a key role in many areas of science and engineering hence an extension to these values is presented. For a function given a simple form of weighted local averaging operator (WLAO) taking into account the particular role of boundary values of the function is constructed. Remarkable features of WLAO was that it contains a parameter of boundary regulation $p$ and depends on a local value $h$ of the integration domain, and WLAO coincides with conventional averaging operator (CAO) at three specific values of $h$, namely $h=0 ; 0.5$ and 1 . By varying these two parameters one can regulate the obtained approximate solutions in order to get more accurate ones. In particular by putting $p=0$, WLAO leads to CAO. It has been shown that the connection of WLAO with Galerkin method forms an effective approximate tool for the buckling problem of columns and the frequency analysis of strongly nonlinear systems. Detailed numerical calculations are carried out with three specific values of the boundary regulation parameter, namely, $p=0.25,0.5$ and 1 for some typical columns and 
strongly nonlinear systems. It is obtained that the accuracy of solutions obtained by WLA is significantly improved in comparison to the one of solutions obtained by CA, specially, when the change of the cross-section or the system nonlinearity are increased. WLA shows to be an effective tool which is sophisticate and can be supported to CA to obtain more accurate solutions. Further comprehensive investigations, however, need to be carried out in order to find appropriate values of the boundary regulation parameter $p$ that can give most approximate solutions for large classes of problems. The key to the best accuracy of obtained solutions is of course related to the best choice of $p$ which will likely depend on the problem to be solved, including the boundary conditions. To solve this problem the approach of the finite element analysis can be useful. It is possible for example, to distinguish the essential boundary conditions from natural boundary conditions Bathe [34], and would use a finer mesh or "overlapping finite elements" at the boundaries with natural boundary conditions Zhang and Bathe [35]. On the other hand the proposed WLA needs to be verified for buckling problems of more complicate structures such as nonlinear columns, laminated composite plates and shells Reddy [36] where the analytical use of Galerkin method is quite often limited by the first order approximation.

\section{ACKNOWLEDGMENTS}

The authors sincerely appreciate valuable comments of Prof. K.J. Bathe which help for further investigation. The paper is supported by Vietnam Academy of Science and Technology under grant number NVCC03.01/20-20 and grant number VAST01.09/20-21.

\section{REFERENCES}

[1] J. T. Oden and J. N. Reddy. Variational methods in theoretical mechanics. Springer-Verlag, NY, (1976).

[2] J. N. Reddy. Applied functional analysis and variational methods in engineering. Krieger Publishing Company, (1991).

[3] J. N. Reddy. An introduction to continuum mechanics with applications. Cambridge University Press, (2008).

[4] J. N. Reddy. Principles of continuum mechanics: A study of conservation principles with applications. Cambridge University Press, (2010).

[5] J. N. Reddy. Energy principles and variational methods in applied mechanics. John Wiley \& Sons, (2017).

[6] J. T. Oden and J. N. Reddy. An introduction to the mathematical theory of finite elements. Wiley, (1976).

[7] J. N. Reddy. An introduction to the finite element method. McGraw-Hill Education, (2005).

[8] C. M. Wang, C. Y. Wang, and J. N. Reddy. Exact solutions for buckling of structural members. CRC Press, (2005).

[9] I. Elishakoff, A. P. Ankitha, and A. Marzani. Rigorous versus naïve implementation of the Galerkin method for stepped beams. Acta Mechanica, 230, (11), (2019), pp. 3861-3873. https://doi.org/10.1007/s00707-019-02393-z.

[10] R. T. Fenner and J. N. Reddy. Mechanics of solids and structures. Second edition, CRC Press, (2012).

[11] L. Euler. Die altitudine colomnarum sub proprio pondere corruentium. Acta Academiae Scientiarum Imperialis Petropolitanae, (1778). (in Latin).

[12] V. P. Agrwal and H. H. Denman. Weighted linearization technique for period approximation in large amplitude non-linear oscillations. Journal of Sound and Vibration, 99, (4), (1985), pp. 463-473. https://doi.org/10.1016/0022$460 x(85) 90534-6$.

[13] Y. K. Cheung, S. H. Chen, and S. L. Lau. A modified Lindstedt-Poincaré method for certain strongly non-linear oscillators. International Journal of Non-Linear Mechanics, 26, (3-4), (1991), pp. 367-378. https://doi.org/10.1016/0020-7462(91)90066-3.

[14] H. S. Y. Chan, K. W. Chung, and Z. Xu. A perturbation-incremental method for strongly non-linear oscillators. International Journal of Non-Linear Mechanics, 31, (1), (1996), pp. 59-72. https://doi.org/10.1016/00207462(95)00043-7.

[15] J. Cai, X. Wu, and Y. P. Li. An equivalent nonlinearization method for strongly nonlinear oscillations. Mechanics Research Communications, 32, (5), (2005), pp. 553-560. https://doi.org/10.1016/j.mechrescom.2004.10.004.

[16] J.-H. He. Homotopy perturbation technique. Computer Methods in Applied Mechanics and Engineering, 178, (3-4), (1999), pp. 257-262. https://doi.org/10.1016/s0045-7825(99)00018-3.

[17] J.-H. He. Some asymptotic methods for strongly nonlinear equations. International Journal of Modern Physics B, 20, (10), (2006), pp. 1141-1199. https://doi.org/10.1142/s0217979206033796.

[18] R. E. Mickens. Truly nonlinear oscillations: harmonic balance, parameter expansions, iteration, and averaging methods. World Scientific, (2010).

[19] N. D. Anh, N. Q. Hai, and D. V. Hieu. The equivalent linearization method with a weighted averaging for analyzing of nonlinear vibrating systems. Latin American Journal of Solids and Structures, 14, (9), (2017), pp. 17231740. https://doi.org/10.1590/1679-78253488. 
[20] H.-L. Zhang. Periodic solutions for some strongly nonlinear oscillations by He's energy balance method. Computers $\mathcal{E}$ Mathematics with Applications, 58, (11-12), (2009), pp. $2480-2485$. https://doi.org/10.1016/j.camwa.2009.03.068.

[21] H.-M. Liu. Approximate period of nonlinear oscillators with discontinuities by modified Lindstedt-Poincare method. Chaos, Solitons E Fractals, 23, (2), (2005), pp. 577-579. https://doi.org/10.1016/j.chaos.2004.05.004.

[22] M. Rafei, D. D. Ganji, H. Daniali, and H. Pashaei. The variational iteration method for nonlinear oscillators with discontinuities. Journal of Sound and Vibration, 305, (4-5), (2007), pp. 614-620. https://doi.org/10.1016/j.jsv.2007.04.020.

[23] S.-Q. Wang and J.-H. He. Nonlinear oscillator with discontinuity by parameter-expansion method. Chaos, Solitons E Fractals, 35, (4), (2008), pp. 688-691. https://doi.org/10.1016/j.chaos.2007.07.055.

[24] N. A. Khan, M. Jamil, and A. Ara. Multiple-parameter Hamiltonian approach for higher accurate approximations of a nonlinear oscillator with discontinuity. International Journal of Differential Equations, 2011, (2011), Article ID 649748. https://doi.org/10.1155/2011/649748.

[25] B. S. Wu, W. P. Sun, and C. W. Lim. An analytical approximate technique for a class of strongly non-linear oscillators. International Journal of Non-Linear Mechanics, 41, (6-7), (2006), pp. 766-774. https://doi.org/10.1016/j.ijnonlinmec.2006.01.006.

[26] R. E. Mickens. Mathematical and numerical study of the Duffing-harmonic oscillator. Journal of Sound and Vibration, 244, (3), (2001), pp. 563-567. https://doi.org/10.1006/jsvi.2000.3502.

[27] C. W. Lim and B. S. Wu. A new analytical approach to the Duffing-harmonic oscillator. Physics Letters A, 311, (4-5), (2003), pp. 365-373. https://doi.org/10.1016/s0375-9601(03)00513-9.

[28] S. B. Tiwari, B. Nageswara Rao, N. Shivakumar Swamy, K. S. Sai, and H. R. Nataraja. Analytical study on a Duffing-harmonic oscillator. Journal of Sound and Vibration, 285, (4-5), (2005), pp. 1217-1222. https://doi.org/10.1016/j.jsv.2004.11.001.

[29] C. W. Lim, B. S. Wu, and W. P. Sun. Higher accuracy analytical approximations to the Duffing-harmonic oscillator. Journal of Sound and Vibration, 296, (4-5), (2006), pp. 1039-1045. https://doi.org/10.1016/j.jsv.2006.02.020.

[30] H. Hu. Solutions of the Duffing-harmonic oscillator by an iteration procedure. Journal of Sound and Vibration, 298, (1-2), (2006), pp. 446-452. https://doi.org/10.1016/j.jsv.2006.05.023.

[31] T. Ozis and A. Yildirim. Determination of the frequency-amplitude relation for a Duffing-harmonic oscillator by the energy balance method. Computers and Mathematics with Applications, 54, (7), (2007), pp. $1184-1187$. https://doi.org/10.1016/j.camwa.2006.12.064.

[32] A. Beléndez, D. I. Méndez, E. Fernández, S. Marini, and I. Pascual. An explicit approximate solution to the Duffing-harmonic oscillator by a cubication method. Physics Letters A, 373, (32), (2009), pp. $2805-2809$. https://doi.org/10.1016/j.physleta.2009.05.074.

[33] M. Bayat, I. Pakar, and G. Domairry. Recent developments of some asymptotic methods and their applications for nonlinear vibration equations in engineering problems: A review. Latin American Journal of Solids and Structures, 9, (2), (2012), pp. 1-93. https://doi.org/10.1590/s1679-78252012000200003.

[34] K.-J. Bathe. Finite element procedures. Second edition, by Klaus-Jurgen Bathe, (2014).

[35] L. Zhang and K.-J. Bathe. Overlapping finite elements for a new paradigm of solution. Computers E Structures, 187, (2017), pp. 64-76. https://doi.org/10.1016/j.compstruc.2017.03.008.

[36] J. N. Reddy. Mechanics of laminated composite plates and shells: theory and analysis. Second edition, CRC Press, (2004). 